EXAMINATION OF THE TEETH OF CHILDREN IN THE PUBLIC SCHOOLS, WITH A REPORT UPON THE EXAMINATION OF 700 PUPILS OF THE PIERCE GRAMMAR SCHOOL, BROOKLINE, MASS.*

\author{
BY WILliaM H. POTTER, D.M.D., \\ Professor of Operative Dentistry, Harvard Dental School.
}

The examination of the teeth of children in the public schools has as yet been carried out in but few places. Such an examination was in force in the city of Strassburg, Germany, in the year 1901, and in the city of Andover, Mass., in the same year. The desirability of such examination is a growing conviction in the minds of those who are giving most thought to the subject, and the reasons for this conviction are as follows:

1. Where examinations have been made a large number of defective teeth have been found.

2. Defective teeth cause the child much pain which interferes with general health and with the ability to study.

3. Defective teeth limit the child's ability to properly masticate food; this leads to indigestion and deficient nutrition. The physical progress of the body is retarded and the mental also.

4. An examination of the teeth gives the opportunity for advice as to necessary treatment and as to prophylactic care.

As a matter of special interest to this meeting I will describe in detail the examination of the teeth of the children of the Pierce School of the town of Brookline, which was undertaken in May and June of this year. I had the pleasure of organizing and directing this work. Dr. D. F. Spinney, Dr. E. N. Kent and Dr. E. B. Wyman, of Brookline, were associated with me and made all the examinations. In this examination the teeth of the children were divided into three classes namely, good, fair, poor. We called the teeth good which had no readily observable defects. Teeth were called fair which presented a few not very serious defects. Teeth were called poor which had several serious defects or many small defects. In order to facilitate the recording of defects there was employed a diagrammatic representation of the teeth of the upper and lower jaws.in which each tooth bore a number and each surface was also numbered. Thus one or more defects in a given tooth could be quickly recorded by the use of a large numeral to indicate the tooth in question and one or more small numbers placed in the position of exponents; these small numbers indicated the surface or surfaces of the given tooth which were defective. For each examining dentist there was appointed a pupil to record the results of the examination. By calling off a series of numbers to the recorder the examiner could quickly register the defects in a given case. In this way the examiner's time was economized in that none of his time was wasted in clerical work.

Besides having a recorder for each examiner we had a person to wash and sterilize instruments. This part of the work was very carefully attended to. There were furnished plenty of boilable mouth mirrors and examination points and each instrument was carefully washed and boiled after each use. With this accurate subdivision of work and economy of time three examiners were enabled to examine in all about fifty pupils in an hour.

So much as to the detail of the work. As to the results of the examination they were as follows: Number of pupils examined, 700; teeth found in good condition, 166, 23\%; teeth found in fair condition, $179,25 \%$; teeth found in poor condition, $355,50 \%$.

These results will bear out the statement which I made in the beginning, namely, that where examinations have been made a large number of defective teeth have been found. In order to turn this examination to a practical account the following card was issued to the pupils examined:

Office of School Committee, Brookline, Mass. NOTICE TO PARENTS.

$\ldots \ldots \ldots \ldots \ldots \ldots \ldots$ has been examined by me and found to have teeth, which need attention. You are advised to take h to your family dentist for further examination and for treatment. If circumstances do not permit consulting a dentist at his office, the child may be treated at a nominal cost at, the Harvard Dental School Infirmary, North Grove Street, Boston, or at the Tufts College Dental School Infirmary, 1416 Huntington Avenue, Boston.

$$
\text { (over) }
$$

DIRECTIONS FOR THE CARE AND USE OF THE TEETH.

1. The teeth should be thoroughly brushed after each meal.

2. A tooth powder used on the brush helps to clean the teeth.

3. Candy and crackers should not be eaten between meals; they cause the teeth to decay.

4. The slow and thorough chewing of hard food helps to preserve the teeth and keep the mouth in a healthy condition.

5. Children's teeth should be examined by a dentist at least twice a year.

To pupils whose teeth were called "good" a card was given on which was printed the "Directions for the care and use of the teeth only."

If examinations of the teeth as here described can accomplish an improved condition of the teeth of the children in the public schools, its value will not, I believe, be questioned by any one. Sound teeth, good digestion, good nutrition and general good health usually go together, while poor teeth, poor digestion, under nutrition and development usually go together.

Here let me refer to one of the best articles which I know of in regard to the examination of the teeth of school children. It was written by Mr. G. E. Johnson, Superintendent of Public Schools, Andover, Mass., and entitled "The Condition of the Teeth of Children in Public Schools," and printed in the International Dental Journal, July, 1901.

In speaking of data which he had collected he says:

"The weight of boys with good teeth was compared with the weight of boys with poor teeth at each age from five to fourteen, also the weight of girls with good teeth with the weight of 
girls with poor teeth of corresponding ages, there being twenty groups in all. The children with good teeth surpassed children with poor teeth in weight in fifteen out of twenty groups.

"Combining the averages of children with good teeth for all ages and the averages of children with poor teeth for all ages, the children with good teeth surpassed the children with poor teeth in weight by an average of 2.7 pounds per child."

I also wish to call attention to an article published in the Deutsche Monatsschrift für Zahnheilkunde 6 March, 1901, by Dr. Ernst Jessen, in which he describes the system established in the city of Strassburg, Germany, by ·which 16,000 of the school children of that eity had a yearly dental examination and were treated in the dental department of the University of Strassburg. Of this article I wrote a synopsis and it was printed in the International Dental Journal, July, 1901.

There are several conclusions which I would make in regard to this work.

1. I believe it is very desirable that once a year the teeth of all children in grammar schools be examined and their condition reported to parents with appropriate advice.

2. This examination should, preferably, be made in the fall of the year soon after the opening of school. At this time the infirmaries of the Dental Schools can best take care of such pupils whose circumstances do not allow them to consult a dentist at his office.

3. Short, practical talks to school children would help much to increase their intelligence as to the value of good teeth in promoting nutrition and general health, and would interest them in the condition of their own mouths. These talks should be given by a dentist, but much supplementary work of the same sort could well be given by the teacher.

\section{A REPORT ON THE EXAMINATION OF THE EYES OF 420 SCHOOL CHILDREN IN THE TOWN OF BROOKLINE.*}

\author{
BY ROBERT G. LORING, M.D. \\ Clinical Assistant, Massachusetts Charitable Eye and Ear
Infirmary.
}

I HAVE to present a report on my examinations of the eyes of some of the school children in the town of Brookline. My work was done at the Pierce School, and I examined four hundred and twenty children, including all grades above the primary. The object of this examination was to find out the number of children with defective eyesight, how many of these could be helped, and how many were, therefore, in need of further examination and treatment. Incidentally, some interesting data were collected with reference to the relation between poor eyesight and poor scholarship in school children.

The work was done necessarily under some difficulties, as more apparatus is required for the most certain results than ordinarily can be

* Read before the Brookline Medical Club Nov. 7, 1906. carried and set up in the schoolhouse. For the careful determination of refractive errors in children, relaxation of the accommodation is of course necessary, and sometimes it is only with a mydriatic that any errors of refraction can be demonstrated. Nevertheless, in the majority of cases all marked degrees of farsightedness, nearsightedness, or astigmatism can be readily discovered, and careful questioning of the child will show the presence or absence of symptoms pointing to eye strain.

The method pursued was as follows: Each child was carefully tested for vision with the ordinary vision card for his ability to read small letters across the room, and was questioned as to the presence of headache, pain in the eyes, or discomfort during or after study. The teacher was asked to state whether the child held the book too near in reading or made any complaint about his eyes. Ability to read the small letters across the room is not, however, a proof that the eyes are normal. Each child was, therefore, examined by the electric retinoscope in a darkened room. This has not been used up to now in the examination of school children, so far as I know, but it has the great advantage of being a purely objective method, by which the refraction of the eye is measured without relying at all upon the statements of the child. Cards for each child were printed, and on these was written the name and age; the vision, the estimated refraction and such symptoms as were found present, and later the child's mark in scholarship.

In general, I found the children in the Pierce School free from the chronic inflammatory diseases, such as conjunctivitis and blepharitis, which are so commonly found in our large city schools filled with children from the tenement districts where the hygienic conditions are bad. There was no trachoma, no phlyctenular disease, and almost no follicular catarrh and only one case of keratitis.

But of course the point of special interest was the question of refraction, $i$. e., the frequency of far- and near-sightedness and astigmatism. I found that 19 out of the 420 children were already wearing glasses, but it appeared from the examination that many more were in urgent need of some relief, either from poor sight or from pains in the eyes and headache.

The children ranged in age from eight to sixteen years, were boys and girls, and included all the grades above the primary. The results were naturally rather hard to tabulate, but in order to make the statistics of practical value, I divided the children into three main classes. In the first class I put all those who saw perfectly on the test card across the room, $i . e$., who had technically $20-20$ of vision, who showed no errors of refraction by the retiniscope, and were not suffering from any symptoms from the use of their eyes. These I called the perfect children, and the first interesting fact was that these were not by any means a majority, in fact less than one half qualified for this class, $i$. e., only 167 out of the 420 children. The remainder 